\title{
Optimal operation points for ultrafast, highly coherent Ge hole spin-orbit qubits
}

\author{
Zhanning Wang $\mathbb{D}^{1,2 凶}$, Elizabeth Marcellina ${ }^{1,2}$, Alex. R. Hamilton $\mathbb{i D}^{1,2}$, James H. Cullen ${ }^{1}$, Sven Rogge $\mathbb{i D}^{1,3}$, Joe Salf ${ }^{4}$ and \\ Dimitrie Culcer (iD $1,2 \otimes$
}

Strong spin-orbit interactions make hole quantum dots central to the quest for electrical spin qubit manipulation enabling fast, lowpower, scalable quantum computation. Yet it is important to establish to what extent spin-orbit coupling exposes qubits to electrical noise, facilitating decoherence. Here, taking $\mathrm{Ge}$ as an example, we show that group IV gate-defined hole spin qubits generically exhibit optimal operation points, defined by the top gate electric field, at which they are both fast and long-lived: the dephasing rate vanishes to first order in the electric field noise along with all directions in space, the electron dipole spin resonance strength is maximized, while relaxation is drastically reduced at small magnetic fields. The existence of optimal operation points is traced to group IV crystal symmetry and properties of the Rashba spin-orbit interaction unique to spin-3/2 systems. Our results overturn the conventional wisdom that fast operation implies reduced lifetimes and suggest group IV hole spin qubits as ideal platforms for ultra-fast, highly coherent scalable quantum computing.

npj Quantum Information (2021)7:54; https://doi.org/10.1038/s41534-021-00386-2

\section{INTRODUCTION}

Quantum computing architectures require reliable qubit initialization, robust single-qubit operations, long coherence times, and a clear pathway towards scaling up. Solid-state platforms are supported by the well-developed solid-state device industry, with mature microfabrication and miniaturization technologies. Among solid-state platforms, semiconductor quantum dot (QD) spin qubits have been actively pursued ${ }^{1}$, with an energetic recent focus on hole spins in diamond and zincblende nano-structures ${ }^{2-26}$.

The primary motivation for this focus is the strong hole spinorbit interaction, which enables qubit control via electron dipole spin resonance (EDSR), making quantum computing platforms faster, more power-efficient, and easier to operate ${ }^{27-34}$. Electric fields are much easier to apply and localize than magnetic fields used in electron spin resonance. Only a global static magnetic field is required to split the qubit levels. In addition, the $p$ symmetry of the hole wave function causes the contact hyperfine interaction to vanish, and complications involving valley degrees of freedom are absent ${ }^{35-39}$. Initial studies indicate that hole spins may possess sufficiently long coherence times for quantum computing ${ }^{40-44}$. Meanwhile, much progress has been made in the initialization and readout of hole spin qubits $8,11,14,17,45,46$

The existential question that will determine the future of hole QD spin qubits is: Does the strong spin-orbit interaction that allows fast qubit operation also enhance undesired couplings to stray fields such as phonons and charge noise leading to intractable relaxation and dephasing? In this paper, we demonstrate theoretically that this is emphatically not the case for hole spin qubits in group IV materials taking Ge as the most prominent example.

In fact, the unique properties of the hole Rashba interaction overturn the conventional understanding of qubit coherence in spin-orbit coupled systems, which states that, as long as the qubit is described by an effective $2 \times 2$ Hamiltonian, holes behave in the same way as electrons. That is, given that the EDSR rate is linear in the spin-orbit strength, while the relaxation and dephasing rates increase as the square of the spin-orbit strength, the Rabi ratio can be enhanced by operating the qubit at points at which the spinorbit interaction is weaker ${ }^{27,28}$. In contrast group IV hole qubits achieve the best coherence when the electrical driving rate is at its maximum. In all other systems, one has to choose between creating long-lived qubits by isolating them from the environment and accepting slower gate times, or designing fast qubits strongly coupled to the environment, but which decohere rapidly.

The key realization is that holes in group IV materials are qualitatively different from group III-V materials. They have tremendous potential for qubit coherence, with $\mathrm{Ge}$ and $\mathrm{Si}$ possessing isotopes with no hyperfine interaction, as well as a near-inversion symmetry that eliminates piezo-electric phonons. This near-inversion symmetry will eliminate the Dresselhaus interaction, the interface inversion asymmetry terms are expected to be negligible in the system ${ }^{47,48}$. Which enables spin resonance in group III-V materials ${ }^{27}$. On the other hand, we show that strong cubic-symmetry terms enable a fully-tunable Rashba coupling. Unlike the Dresselhaus interaction, and unlike electron systems, the hole Rashba interaction evolves nonlinearly as a function of the gate electric field, a fact traced to the hole spin-3/2. The qualitative difference between the Rashba and Dresselhaus interactions for holes is vital for qubit coherence. Thanks to this nonlinearity, dephasing due to electric field fluctuations in all spatial directions can be essentially eliminated at specific optimal operation points defined by the gate electric field ${ }^{15,16,38,49-54}$. At these points electrical qubit rotations are at their most efficient, with the spin resonance Rabi gate time at a minimum. The relaxation rate due to phonons can be made as small as desired at small magnetic fields of the order of $0.1 \mathrm{~T}$, which allows $10^{6}-10^{7}$

\footnotetext{
${ }^{1}$ School of Physics, The University of New South Wales, Sydney, NSW, Australia. ${ }^{2}$ Australian Research Council Centre of Excellence in the Future Low-Energy Electronics Technologies, The University of New South Wales, Sydney, NSW, Australia. ${ }^{3}$ Center for Quantum Computation and Communication Technology, The University of New South Wales, Sydney, NSW, Australia. ${ }^{4}$ Department of Electrical and Computer Engineering, University of British Columbia, Vancouver, BC, Canada. ${ }^{\circledR}$ email: zhanning.wang@unsw.edu. au; d.culcer@unsw.edu.au
} 


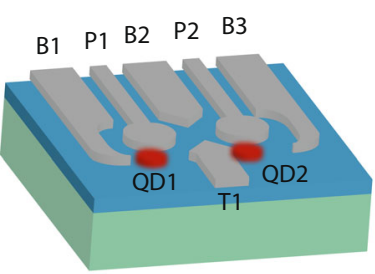

Fig. 1 A prototype double quantum dot in a 2D hole gas. The red shaded circles represent two quantum dots confined by a set of gates. Our focus is on a single dot; two dots are shown to illustrate scaling up strategies, e.g., gates $B_{2}$ and $T_{1}$ control inter-dot tunneling.

operations in one relaxation time for an in-plane alternating field $E_{\mathrm{AC}} \sim 10^{3} \mathrm{Vm}^{-1}$.

Our focus in this paper is on Ge, which has witnessed enormous recent progress $6,7,9,10,55$. Holes in planar Ge quantum wells have a very large out of plane Landé g-factor, $g \approx 20$, enabling operation at very small magnetic fields, which would not impede coupling to a superconducting resonator. The low resistivity of $\mathrm{Ge}$ when contacting with metals makes couplings between other devices such as superconductors easier ${ }^{9,56,57}$. In the past decade some spectacular results have been reported, for example, EDSR detection techniques ${ }^{18,55}$, structures of quantum confinement systems $s^{7,58-60}$, the anisotropy of $g$-tensors ${ }^{10,58}$, spin-orbit couplings, and transport phenomena in two-dimensional hole systems ${ }^{4,6,10,61,62}$. We focus a on single dot throughout this work. A prototype device, including a neighboring dot, is shown in Fig. 1. The Hamiltonian describing a single hole quantum dot has the general form $\mathrm{H}=\mathrm{H}_{\mathrm{LK}}+H_{\mathrm{BP}}+H_{\mathrm{Z}}+H_{\mathrm{ph}}+H_{\text {conf, }}$ where $H_{\mathrm{LK}}$ represents the Luttinger-Kohn Hamiltonian, $H_{Z}$ is the Zeeman interaction between the hole and an external magnetic field, and $H_{\mathrm{ph}}$ the hole-phonon interaction. $H_{\text {conf }}$ is the confinement potential including the vertical and lateral confinement. The vertical confinement is achieved by applying a gate electric field $F_{z}$ in the growth direction, leading to a term $e F_{z} z$ in the Hamiltonian; the lateral confinement is modeled as an in-plane parabolic potential well. The Bir-Pikus Hamiltonian $H_{\mathrm{BP}}$ represents strain ${ }^{18}$, which appears naturally as part of the quantum well growth process. A typical configuration of holes in $\mathrm{Ge}$ is achieved by growing a thin strained Ge layer (usually about $10 \mathrm{~nm}$ to $20 \mathrm{~nm}$ ) between SiGe layers such that, if the barrier between the two layers is high enough, a quantum well can be formed. We consider $\mathrm{Si}_{x} \mathrm{Ge}_{1-x}$, where $x=0.15^{4,6,7,58}$, with other values of $x$ discussed in Supplementary Table 1.

We start from the bulk band structure of holes as derived by Luttinger and $\mathrm{Kohn}^{63}$. The spinor basis is formed by the eigenstates of $J_{z},\left\{\left|+\frac{3}{2}\right\rangle,\left|-\frac{3}{2}\right\rangle,\left|+\frac{1}{2}\right\rangle,\left|-\frac{1}{2}\right\rangle\right\}$. For a 2D hole gas grown along $\hat{z} \|$ (001), we write the Luttinger-Kohn Hamiltonian as:

$$
H_{\mathrm{LK}}\left(k^{2}, k_{\mathrm{z}}\right)=\left[\begin{array}{cccc}
P+Q & 0 & L & M \\
0 & P+Q & M^{*} & -L^{*} \\
L^{*} & M & P-Q & 0 \\
M^{*} & -L & 0 & P-Q
\end{array}\right],
$$

where $P=\frac{\hbar^{2} \gamma_{1}}{2 m_{0}}\left(k^{2}+k_{\mathrm{z}}^{2}\right), Q=-\frac{\hbar^{2} \gamma_{2}}{2 m_{0}}\left(2 k_{\mathrm{z}}^{2}-k^{2}\right), L=-\sqrt{3} \frac{\hbar^{2} \gamma_{3}}{2 m_{0}} k_{-} k_{\mathrm{z}}$ $M=-\frac{\sqrt{3} \hbar^{2}}{2 m_{0}}\left(\bar{\gamma} k_{-}^{2}+\delta k_{+}^{2}\right)$, and $m_{0}$ is the free electron mass, $\gamma_{1}, \gamma_{2}, \gamma_{3}$ are Luttinger parameters which are determined by the band structure. The in-plane wave vector will be $k^{2}=k_{x}^{2}+k_{y}^{2}, k_{ \pm}=k_{x} \pm$ $i k_{y}$. In this manuscript, we use the symmetric gauge $\mathbf{A}=(B / 2)(-y, x$, $0)$ so that $\mathbf{k} \rightarrow-i \nabla+e \mathbf{A} / \hbar$. We have also used $\bar{\gamma}=\left(\gamma_{2}+\gamma_{3}\right) / 2$ and $\delta=\left(\gamma_{3}-\gamma_{2}\right) / 2$ to simplify the algebra. In Ge $\delta / \bar{\gamma}<0.15$, hence $\delta$ can be treated perturbatively, while bulk Dresselhaus terms are absent. Although interface inversion asymmetry terms with the same functional form may exist ${ }^{47}$, at the strong gate fields considered here will be overwhelmed by the Rashba interaction and are not discussed in detail. The diagonal terms of $H_{\mathrm{BP}}$ in the $\mathrm{HH}$ manifold are $P_{\varepsilon}+Q_{\varepsilon}=-a_{\mathrm{v}}\left(\varepsilon_{x x}+\varepsilon_{y y}+\varepsilon_{z z}\right)$, while in the LH manifold they are $P_{\varepsilon}-Q_{\varepsilon}=-\left(b_{\mathrm{v}} / 2\right)\left(\varepsilon_{\mathrm{xx}}+\varepsilon_{\mathrm{yy}}-2 \varepsilon_{\mathrm{zz}}\right)$, where $a_{\mathrm{v}}=-2 \mathrm{eV}$ and $b_{\mathrm{v}}=-2.3 \mathrm{eV}$ are deformation potential constants ${ }^{18}$. In our chosen configuration $\varepsilon_{\mathrm{xx}}=\varepsilon_{\mathrm{yy}}=-0.006$, the minus sign indicates that the germanium is compressed in $x y$-plane. In the $\hat{z}$-direction, the $\mathrm{Ge}$ layer will be stretched, and $\varepsilon_{\mathrm{zz}}=\left(-2 C_{12} / C_{11}\right) \varepsilon_{x x}=0.0042$, with $C_{12}=44 \mathrm{GPa}, C_{11}=126 \mathrm{GPa}$ for $\mathrm{Ge}$. The diagonal terms of the strain-relaxed barrier configuration will change the $\mathrm{HH}-\mathrm{LH}$ energy splitting by a constant, which is approximately $50 \mathrm{meV}$.

The growth direction provides the spin quantization axis, with the heavy hole states ( $\mathrm{HHs}$ ) representing the $\pm 3 / 2$ angular momentum projection onto this axis, while the light hole states (LHs) represent $\pm 1 / 2$. In $2 \mathrm{D}$ hole systems, the $\mathrm{HHs}$ are the ground state $^{7,27,51,64,65}$. To define a quantum dot a series of gates are added on top of the 2D hole gas confinement, as in Fig. 1, and we ultimately seek an effective Hamiltonian describing the two lowest-lying $\mathrm{HH}$ states in a quantum dot. Since we expect the $\mathrm{HH}$ LH splitting to be much larger than the quantum dot confinement energy, we proceed with the standard assumptions of $\mathbf{k} \cdot \mathbf{p}$ theory, retaining at first only terms containing $k_{\mathrm{z}}$, with $k_{\mathrm{x}}$ and $k_{\mathrm{y}}$ initially set to zero. This determines the approximate eigenstates $\psi_{\mathrm{H}, \mathrm{L}}(z)$ corresponding to the growth-direction. These are described by two variational Bastard wave functions $\psi_{\mathrm{H}}$ and $\psi_{\mathrm{L}}{ }^{48,66}$,

$\psi_{\mathrm{H}, \mathrm{L}}(z)=\sqrt{\frac{4 \beta_{\mathrm{H}, \mathrm{L}}\left(\pi^{2}+\beta_{\mathrm{H}, \mathrm{L}}^{2}\right)}{\left(1-e^{\left.-2 \beta_{\mathrm{H}, \mathrm{L}}\right) d \pi^{2}}\right.}} \cos \left(\frac{\pi z}{d}\right) \exp \left[-\beta_{\mathrm{H}, \mathrm{L}}\left(\frac{z}{d}+\frac{1}{2}\right)\right]$,

where the dimensionless variational parameters $\beta_{\mathrm{H}, \mathrm{L}}$ are sensitive to the gate electric field due to the term $e F_{z} z$, and $d$ is the width of the quantum well in the growth direction, which is an input parameter. The orthogonality of the $\mathrm{HH}$ and $\mathrm{LH}$ states is ensured by the spinors. This wave function is suitable for inversion layers, as well as accumulation layers, although our focus will be primarily on the latter. For inversion layers, the Bastard wave function will also be appropriate, because in experiments the electric field can be made large enough such that the hole gas sticks around the top of the quantum well.

In the $x y$-plane, we model the confinement potential using a harmonic oscillator

$$
\left[\frac{\hbar^{2}}{2 m_{p}}\left(-i \nabla_{\|}+e \mathbf{A}\right)^{2}+\frac{1}{2} m_{\mathrm{p}} \omega_{0}^{2}\left(x^{2}+y^{2}\right)\right] \phi=\varepsilon \phi
$$

where $m_{\mathrm{p}}=m_{0} /\left(\gamma_{1}+\gamma_{2}\right)$ is the in-plane effective mass of the heavy holes, the subscript $\|$ refers to the $x y$-plane, $\omega_{0}$ is the oscillator frequency, $a_{0}$ the QD radius which satisfies, $a_{0}^{2}=\hbar /\left(m_{\mathrm{p}} \omega_{1}\right)$ i.e., a magnetic field will narrow the QD radius. The solutions are the well-known Fock-Darwin wave functions $\left|\phi_{n_{1}, n_{2}}\right\rangle^{18}$ with eigenenergies $\quad \varepsilon_{n_{1}, n_{2}}=\hbar\left(n_{1}+n_{2}+1\right) \omega_{l}+\frac{1}{2} \hbar\left(n_{2}-n_{1}\right) \omega_{c}$, where $\omega_{l}=\sqrt{\omega_{0}^{2}+\omega_{c}^{2} / 4}, \omega_{\mathrm{c}}=e B / m_{\mathrm{p}}$ is the cyclotron frequency. The Bastard wave functions account for the perpendicular confinement, in the $\hat{\mathbf{z}}$-direction. The Fock-Darwin wave functions $\left|\phi_{n_{1}, n_{2}}\right\rangle$ account for the quantum dot confinement in the $x y$-plane. The Bastard and Fock-Darwin wave functions would be formally the same for electrons, while in a hole gas is that separate Bastard wave functions are required for the heavy and light holes.

Finally, the hole-phonon interaction is ${ }^{67-69}$ :

$H_{\mathrm{i}, \mathrm{j}, \mathrm{s}}=\sum_{a, \beta=\mathrm{x}, \mathrm{y}, \mathrm{z}} \frac{1}{2} \sqrt{\frac{\hbar}{2 N V_{\mathrm{c}} \rho \omega_{\mathrm{s}}}} D_{\alpha, \beta}^{\mathrm{i}, \mathrm{j}}\left[\frac{q_{\alpha} \hat{e}_{\mathrm{s}, \beta}+q_{\beta} \hat{e}_{\mathrm{s}, \alpha}}{q}\right] q\left(e^{-i \mathbf{q} \cdot \mathbf{r}} \hat{a}_{q}^{\dagger}+e^{i \mathbf{q} \cdot \mathbf{r}} \hat{a}_{q}\right)$,

where $q$ is the phonon wave vector, $V_{c}$ is the unit cell volume, $N V_{c}$ is the crystal volume, $\hat{e}_{s}$ is the polarization direction vector. The 
density of the material is denoted by $\rho, D_{\alpha, \beta}$ represents the deformation potential matrix, and $\hat{a}^{\dagger}$ and $\hat{a}$ are the phonon creation and the annihilation operators. The details are presented in the Supplementary Information Eq. (1) $)^{36,70-74}$.

Our approach is semi-analytical. To incorporate the contributions from the LHs, and the Rashba spin-orbit couplings, we start from the $4 \times 4$ Luttinger Hamiltonian and project it onto the product states of the out-of-plane sub-bands $\mathrm{HH} 1, \mathrm{LH} 1$, each with two spin projections, and the first four orbital levels of the in-plane confinement, such that our Hamiltonian matrix is $40 \times 40$. This refers to states $\left\{\left|\frac{3}{2}, S\right\rangle \otimes\left|\phi_{n, m}\right\rangle\right\}$, where $S=3 / 2,-3 / 2,1 / 2,-1 / 2$ represent the HHs or LHs, $n=0,1,2,3$ and $m=0, \pm 1,2, \pm 3$ denote the in-plane Fock-Darwin states. We have checked that addition of the HH2, LH2 sub-bands does not modify the results, this is attributed to the significant energy gaps separating them from HH1, LH1. Given the high computational cost of adding these subbands, we have not taken them into account in the results presented here. To obtain the matrix elements required for the dephasing and relaxation times, as well as for the EDSR Rabi frequency, we perform a 3rd order Schrieffer-Wolff transformation on the $40 \times 40$ Hamiltonian. This transformation takes into account all the spin-orbit terms that are not separable in the spatial coordinates, which are precisely the terms leading to the Rashba interaction. To ensure the accuracy of the Schrieffer-Wolff method we compare the results for the Larmor frequency with a full numerical diagonalization of the $40 \times 40$ matrix.

\section{RESULTS AND DISCUSSION}

\section{Qubit Zeeman splitting}

The qubit Larmor frequency has been plotted in Fig. 2 as a function of the gate electric field. The Schrieffer-Wolff method agrees well with the numerical diagonalization: the location of the optimal operation point differs by only $2 \%$ in the two approaches. We note the non-monotonic behavior as a function of the gate field, leading to an optimal operation point in the range of $30-50 \mathrm{MV} / \mathrm{m}$. Electric fields of such magnitude are used routinely in quantum computing experiments $7,46,75,76$. The non-monotonic behavior is directly related to the behavior of Rashba spin-orbit coupling discussed below. We note that the spatial dimensionality of the qubit is determined by the relevant energy scales, namely the $\hat{\mathbf{z}}$-sub-band energy spacing compared to the energy splitting

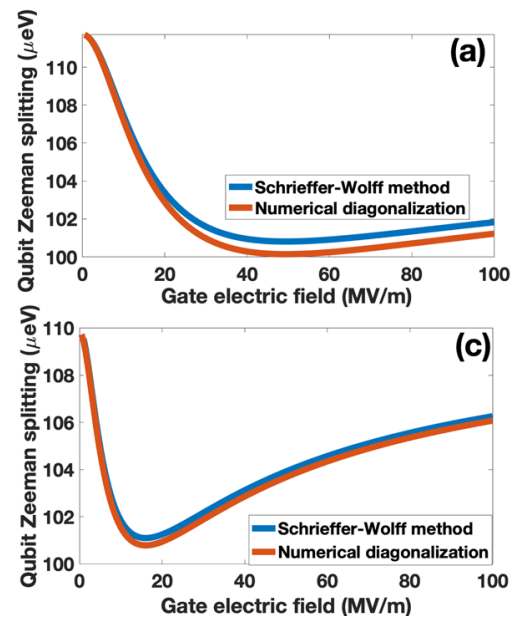

of the lateral wave functions. The heavy hole- light hole splitting, given by the perpendicular confinement, is many times larger than the in-plane qubit confinement energy, determined by the in-plane confinement, so the system is in the quasi-2D limit. Nevertheless, our findings, such as trends with the top gate field, can be interpreted qualitatively by analogy with the Rashba interaction in the asymptotic 2D limit $d \rightarrow 0^{77}$, which we also obtain from Eq. (1). For a system with cubic symmetry this contains two terms with different rotational properties:

$H_{\mathrm{SO}}=i a_{2}\left(k_{+}^{3} \hat{\sigma}_{-}-k_{-}^{3} \hat{\sigma}_{+}\right)+i a_{3}\left(k_{+} k_{-} k_{+} \hat{\sigma}_{+}-k_{-} k_{+} k_{-} \hat{\sigma}_{-}\right)$,

where $\hat{\sigma}_{ \pm} \equiv\left(\hat{\sigma}_{\mathrm{x}} \pm i \hat{\sigma}_{\mathrm{y}}\right) / 2$. The coefficients are evaluated as:

$$
\begin{aligned}
& a_{2}=\frac{3}{2} \frac{\mu^{2} \bar{\gamma} \gamma_{3}}{E_{\mathrm{H}}-E_{\mathrm{L}}}\left\langle\psi_{\mathrm{H}} \mid \psi_{\mathrm{L}}\right\rangle\left[\left\langle\psi_{\mathrm{H}}\left|\hat{k}_{\mathrm{z}}\right| \psi_{\mathrm{L}}\right\rangle-\left\langle\psi_{\mathrm{L}}\left|\hat{k}_{\mathrm{z}}\right| \psi_{\mathrm{H}}\right\rangle\right] \\
& a_{3}=\frac{3}{2} \frac{\mu^{2} \delta \gamma_{3}}{E_{\mathrm{H}}-E_{\mathrm{L}}}\left\langle\psi_{\mathrm{H}} \mid \psi_{\mathrm{L}}\right\rangle\left[\left\langle\psi_{\mathrm{H}}\left|\hat{k}_{\mathrm{z}}\right| \psi_{\mathrm{L}}\right\rangle-\left\langle\psi_{\mathrm{L}}\left|\hat{k}_{\mathrm{z}}\right| \psi_{\mathrm{H}}\right\rangle\right],
\end{aligned}
$$

where $E_{\mathrm{H}}$ and $E_{\mathrm{L}}$ are the energies of the lowest-lying $\mathrm{HH}$ and $\mathrm{LH}$ states, respectively, and are strong functions of the gate electric field. These formulas explain three main features.

Firstly, the optimal operation point reflects the interplay of the quadrupole degree of freedom with the gate electric field unique to spin-3/2 systems. The behavior of the qubit Zeeman splitting and Rashba coefficients is understood by recalling that the Rashba effect for the $\mathrm{HH}$ sub-bands is primarily driven by the off-diagonal matrix element $L$ in Eq.(1) connecting the $\mathrm{HH}$ and $\mathrm{LH}$ sub-bands. This term, which is $\propto k_{z} k_{+}$, increases with the top gate field. At small gate fields, the Rashba spin-orbit constants increase monotonically due to the increase in the $k_{\mathrm{z}}$ overlap integral. This continues until a critical top gate field is reached at which the $\mathrm{HH}-\mathrm{LH}$ splittings, determined by the matrix element $Q$, begin to increase faster than the off-diagonal matrix element $L$. The heavy hole-light hole splitting induced by the confinement potential and the gate electric field is traced to the different effective masses for heavy and light holes. This physics has been shown previously by Winkler and collaborators ${ }^{78-80}$. Beyond this critical field, the Rashba terms decrease, resulting in a relatively broad optimal operation region at which the qubit is insensitive to background electric field fluctuations in the $\hat{z}$-direction and the dephasing rate vanishes to first order in the $\hat{z}$-electric field. As we show below, electric field fluctuations in the $\hat{z}$-direction are by far the most damaging to the qubit and are the key source of decoherence to

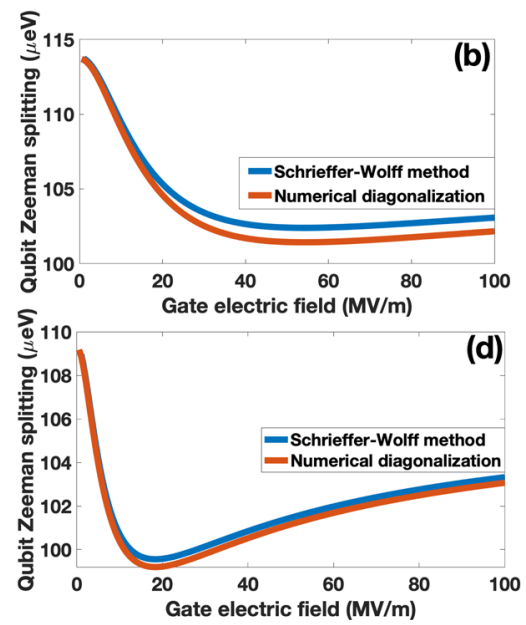

Fig. 2 The qubit Zeeman splitting. Comparison of the qubit Zeeman splitting between Schrieffer-Wolff transformation (to the third order) and exact numerical diagonalization for four different configurations. When the gate electric field is turned off, the qubit Zeeman splitting $g_{\mathrm{o}} \mu_{B} B \approx 110 \mu \mathrm{eV}$. In all these figures, the out-of-plane magnetic field is $B=0.1 \mathrm{~T}$. We can notice that the sweet spot does not change much as a function of the quantum dot radius, but the size of the qubit Zeeman splitting will be smaller for a larger quantum dot size. In all of these plots, we have $\hbar \omega_{l} \gg g_{0} \mu_{B} B$. Numerical diagonalization is the red curve, Schrieffer-Wolff method is the blue curve. a $d=11 \mathrm{~nm}, a_{0}=50 \mathrm{~nm}$. b $d=11 \mathrm{~nm}, a_{0}=60 \mathrm{~nm}$. c $d=15 \mathrm{~nm}, a_{0}=50 \mathrm{~nm}$. $\mathbf{d} d=15 \mathrm{~nm}, a_{0}=60 \mathrm{~nm}$. 
be avoided. The breadth and smoothness of the extreme make the tuning of the electric field to reach the optimal operation point easier.

Secondly, the sweet spot shifts slightly with the dot radius ${ }^{64}$, and this is fully captured by our Schrieffer-Wolff results. The reason for this is that, in a 2D system, while the angular form of the Rashba interaction is dictated by rotational symmetry, the Rashba coupling constants $a_{2}$ and $a_{3}$ are functions of the magnitude of the wave vector. This implies they are functions of the density, and this is vital at large densities. Hence a quantum dot can be envisaged as having Rashba parameters that are functions of the in-plane radius $a_{0}$, and their dependence is less pronounced at larger $a_{0}$, since this corresponds to smaller densities.

Thirdly, each of the two spin-orbit coupling terms in Eq. (5) can be envisaged as the interaction of the hole spin with an effective spin-orbit field that depends on the momentum. In the absence of a magnetic field, the $a_{2}$-Rashba spin-orbit field winds around the Fermi surface three times, whereas the $a_{3}$-Rashba spin-orbit field winds only once. In the strict $2 \mathrm{D}$ limit it is the $a_{3}$-term that enables EDSR. Although the quantum dot is not in the exact 2D limit, it still holds that EDSR is enabled by the cubic symmetry terms $\propto \delta$. Setting $\delta=0$ in our calculations causes the EDSR frequency to vanish.

$f_{\mathrm{EDSR}}=24 g_{0} \mu_{\mathrm{B}} B e E_{\mathrm{AC}} a_{0}^{2} \delta m_{\mathrm{p}}^{2} \times u$

where $u$ are a long expression that can be found in the Supplementary Information Eq. (17).

\section{Dephasing time}

The main dephasing mechanisms are fluctuating electrical fields such as charge noise. We focus on random telegraph noise (RTN) due to charge defects, noting that a similar discussion can be presented for $1 / f$ noise, which is typically caused by an incoherent superposition of RTN sources. For this reason, we expect the trends for the two types of noise to be similar, while reliable numbers for $1 / f$ noise must await the experimental determination of the noise spectral density $S(\omega)$ for hole qubits. To begin with, we estimate the dephasing time $T_{2}^{*}$, which is expected to be primarily determined by fluctuations in the Larmor frequency of the qubit induced by charge noise. The electric potential induced at the qubit by a defect located at $\mathbf{r}_{\mathrm{D}}$, which may give rise to RTN, can be modeled as a quasi-2D screened Coulomb potential:

$U_{\mathrm{sCr}}=\frac{e^{2}}{2 \epsilon_{0} \epsilon_{\mathrm{r}}} \int_{0}^{2 k_{\mathrm{F}}} \frac{e^{-i \mathbf{q} \cdot\left(\mathbf{r}-\mathbf{r}_{\mathrm{D}}\right)}}{q+q_{\mathrm{TF}}} \frac{\mathrm{d}^{q}}{(2 \pi)^{3}}$,

where $\epsilon_{0}$ is the vacuum permeability, $\epsilon_{\mathrm{r}}$ is the relative permeability for $\mathrm{Ge}, q_{\mathrm{TF}}$ is the Thomas-Fermi wave vector, and $k_{F}$ is the Fermi wave vector ${ }^{49}$. In a dilution refrigerator, the high energy modes of the Coulomb potential are negligible, therefore the $q>2 k_{F}$ part is ignored. Another source of dephasing is dipole defects due to the asymmetry in bond polarities.

$U_{\text {dip }}\left(\mathbf{R}_{\mathrm{D}}\right)=\frac{\mathbf{p} \cdot \mathbf{R}_{\mathrm{D}}}{4 \pi \epsilon_{0} \epsilon_{\mathrm{r}} R_{\mathrm{D}}^{3}}$,

where $\mathbf{R}_{\mathrm{D}}$ is the distance between the dot and the unscreened charge dipole. $\boldsymbol{p}$ is the dipole moment of the charge $\mathbf{p}=e \mathbf{l}$, the size of the dipole is about $1 \AA$.

As a worst-case estimate of the dephasing time, we use the motional narrowing result ${ }^{49,50}$, the dephasing time $T_{2}^{*-1}=(\delta \omega)^{2} \tau / 2$, where $\delta \omega$ is the change in qubit Larmor frequency due to the fluctuator, and we consider $\tau=10^{3} t_{\text {Rabi, }}$ where $t_{\text {Rabi }}$ is the single-qubit operation time (the inverse of the EDSR frequency), which can be found from Fig. 3a. Because of the weak coupling between the spin degree of freedom and external reservoirs, slower fluctuators can be eliminated via pulse sequences and the spin-echo techniques ${ }^{81}$. We consider two sample defects separately. One is a single-charge defect located $100 \mathrm{~nm}$ away from the quantum dot in the plane of the dot as a worst-case scenario for a charge trap. We use $r_{D}=100 \mathrm{~nm}$ since regions inside this range will be depleted by the top gate, and charge traps will not be active. We also consider a dipole defect immediately under the gate and above the dot, with $R_{\mathrm{D}}=20 \mathrm{~nm}$ in the $\hat{z}$ direction. This is because within the depleted region the most relevant defects are charge dipoles, whose orientation fluctuates. To estimate the pure dephasing time at the optimal operation point due to such a defect, we first note that the inplane electric field will not contribute to dephasing. An in-plane electric field enters the QD Hamiltonian as $\mathbf{E}_{\|} \cdot \mathbf{r}_{\|}$. This in-plane electric field term does not couple states with different spin orientations. When we consider the qubit Zeeman splittings, the corrections to the effective quantum dot levels due to the in-plane electric field will read the same for $H_{1,1}$ and $H_{2,2}$ up to the secondorder, therefore, fluctuations in qubit Zeeman splitting $H_{1,1}-H_{2,2}$ will not depend on the in-plane electric field. However, higherorder terms in the expansion of the electrostatic potential of the
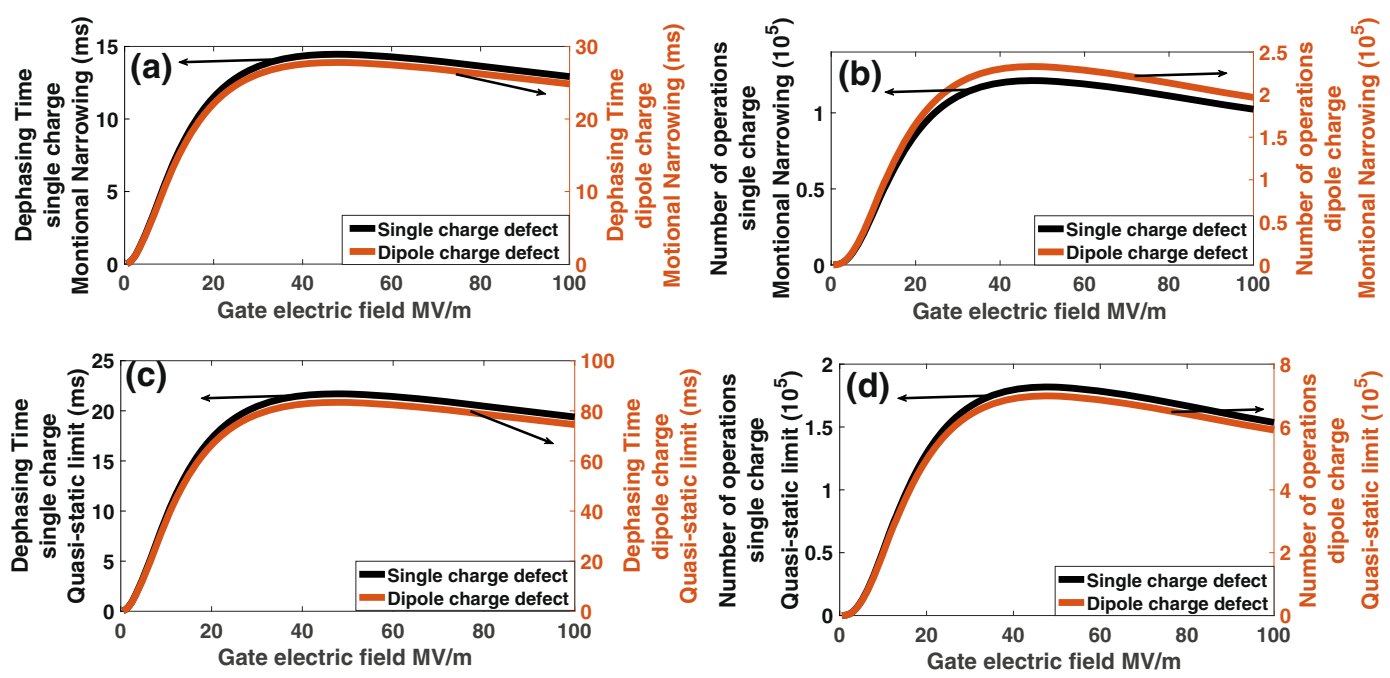

Fig. 3 Dephasing time. In all plots, the quantum well width is $d=11 \mathrm{~nm}$ and dot radius $a_{0}=50 \mathrm{~nm}$. a Dephasing time in the motional narrowing regime. $\mathbf{b}$ The allowable number of single-qubit operations in one dephasing time in motional narrowing regime. $\mathbf{c}$ Dephasing time in the quasi-static limit. $\mathbf{d}$ The allowable number of single-qubit operations in one dephasing time in the quasi-static limit. 
defects will lead to dephasing, and these are responsible for dephasing at the optimal operation point itself. To determine their effect, we write the ground state energy as $E_{\mathrm{LK}}+E_{0}+E_{\mathrm{z}}+v_{0}$ where $E_{0}$ is the lateral confinement energy, $E_{z}$ is the Zeeman energy, and $v_{0}$ is the energy correction due to the defect.

We estimate the approximate qubit window of operation around the optimal operation point. Away from the optimal operation point, due to the fluctuating electric potential of the defect, the energy levels of the quantum dot will gain a correction, i.e., $\left\langle\phi_{n_{1}, m_{1}}\left|U_{s c}\right| \phi_{n_{2}, m_{2}}\right\rangle$. With these assumptions, the dephasing time is plotted as a function of the gate electric field in Fig. 3. At the optimal operation point, the dephasing time due to the outof-plane fluctuations is calculated to the second-order, since the first-order fluctuation vanishes, the in-plane fluctuations will dominate the dephasing. Away from the optimal operation point, the motional narrowing result is much smaller than the quasistatic limit result. This is because the first-order variation of the qubit Zeeman splitting will weaken the correlation time, while the quasi-static limit does not consider any correlations. However, as the gate electric field approaches the optimal operation point, the variation of qubit Zeeman splitting decreases; at the optimal operation point, compared with the quasi-static limit result, a longer correlation time will lead to a larger dephasing time. We also determine the pure dephasing time in the quasi-static limit, where the switching time is the longest time scale in the system. This is essentially given by $T_{2}=2 \pi /(\delta \omega)$, and is plotted in Fig. $3 \mathrm{~b}$.

\section{Relaxation time and EDSR}

We briefly discuss electrically driven spin resonance. An in-plane oscillating electric field represented in the Hamiltonian by $e E_{\mathrm{AC}}(t) x$ drives spin-conserving transitions between the QD states. For a multiple occupied hole dot, the excited state structure may be more complex but the argument above remains valid because the $a_{2}$ and $a_{3}$ Rashba terms couple the ground state to different excited states. The spin resonance Rabi time is the time taken to accomplish an operation. The Rabi frequency can be tuned by changing the gate electric field and with it the Rashba spin-orbit coupling constant. However, note that because the two Rashba terms directly determine the correction to the $g$-factor, the Rashba interaction and the $g$-factor cannot be tuned independently at present.

Given that the spin resonance frequency is a maximum at the optimal operation point, it follows that the qubit can be tuned to have maximum coherence and maximum electrical driving simultaneously. The nonlinearity in the hole Rashba interaction as a function of the gate field that enables this feature has no counterpart in electron systems. In GaAs hole systems ${ }^{27}$ this nonlinearity does not lead to optimal operation points. This is because, firstly, the spin resonance Rabi frequency in GaAs hole qubits is driven by the Dresselhaus interaction, which is not tunable via the gate electric field, while $a_{3}$ is negligible in GaAs. Secondly, GaAs qubits are exposed to decoherence through the hyperfine interaction, piezoelectric phonons, and the Dresselhaus interaction, none of which can be mitigated.

Since the Rabi frequency is maximized at the optimal operation point, the relaxation time $T_{1}$ is minimized there. For the qubit to be operated efficiently it is vital to determine the ratio of the EDSR and relaxation rates. Hyperfine interactions and phonon-hole interactions are two major factors affecting the relaxation time, hence the quality of the qubit. However, the $p$-type symmetry of the valence band excludes the contact hyperfine interaction. There is no bulk inversion asymmetry in group IV elements; this leads to no Dresselhaus spin-orbit coupling. However, there is still the Rashba spin-orbit coupling due to the structure inversion asymmetry, which couples the heavy-hole states to the light-hole states. Neither the spin nor the orbital angular momentum will be a good quantum number, as the admixture of the spin-down and the spin-up states will modify the wave functions. We emphasize that, whereas EDSR comes only from the $a_{3}$-Rashba term, the qubit relaxation is caused by both the $a_{2^{-}}$and the $a_{3^{-}}$ Rashba terms.

The relaxation time evaluated using Fermi's golden rule is shown in Fig. 4. For completeness, we also consider two-phonon relaxation processes, which include virtual emission and absorption of a phonon between two heavy hole states, since in the firstorder relaxation calculation there is no direct matrix element between the two heavy-hole states. However, the two-phonon process calculation returns a negligible relaxation rate, which will not contribute significantly to the relaxation time. The relaxation rate will depend on the external magnetic field as $\left(1 / T_{1}\right) \propto B^{7}$ for the $a_{3}$-Rashba term and $\left(1 / T_{1}\right) \propto B^{9}$ for the $a_{2}$-Rashba term. This is shown in Fig. 4a.

We also plot the ratio between the relaxation time and the EDSR time, demonstrating that the system allows for a large number of operations. The allowable number of single-qubit operations is calculated by evaluating the ratio of the relaxation time and the EDSR time, i.e., the Rabi ratio. The in-plane electric field we used is $E_{\mathrm{AC}}=10^{3} \mathrm{~V} / \mathrm{m}$. In Fig. 4, we plot the relaxation time, EDSR Rabi time, comparison of the magnitude of the relaxation time and EDSR time and an estimation of red the allowable number of single-qubit operations as the function of the gate electric field at
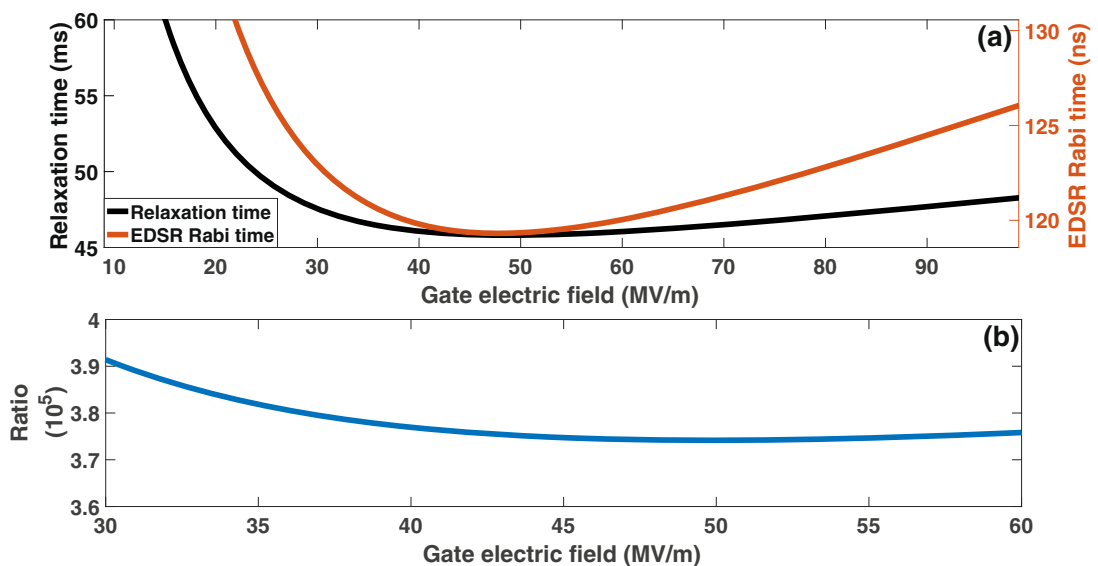

Fig. 4 The relaxation time and the EDSR Rabi time. In all plots $d=11 \mathrm{~nm}, a_{0}=50 \mathrm{~nm}$, the external magnetic field is $B=0.1 \mathrm{~T}$. The density of Ge $\rho=5.33 \times 10^{3} \mathrm{~kg} / \mathrm{m}^{3}$. The phonon propagation speed along the transverse direction is $v_{t}=3.57 \times 10^{3} \mathrm{~m} / \mathrm{s}$, along the longitudinal direction it is $v_{l}=4.85 \times 10^{3} \mathrm{~m} / \mathrm{s}$. a Relaxation time and EDSR Rabi time as a function of the gate electric field. $\mathbf{b}$ The allowable number of single-qubit operations in one relaxation time. 
a magnetic field $B=0.1 \mathrm{~T}$ which is parallel to the growth direction. The relaxation time calculations mainly consider the hole-phonon interactions and the details can be found in the Supplementary Information Eq. (22). Both the relaxation time and the EDSR time will depend on the spin-orbit coupling coefficients, therefore, their extrema coincide. The relaxation time increases for smaller dot sizes because that corresponds to larger confinement energy, while both the phonon and the spin-orbit coupling terms connect the orbital ground state to higher excited states. From Fig. 4a, we can see that the Ge hole quantum dot has a long relaxation time and large Rabi ratio at dilution refrigerator temperatures. It is also useful to study the relaxation time at slightly higher temperatures, e.g., $4 \mathrm{~K}$, at which both phonon absorption and emission must be taken into account. The phonon occupation number is given by the Bose-Einstein distribution $N=\left(e^{\hbar \omega /\left(k_{B} T\right)}-1\right)^{-1}$, where $N$ is the occupation number, $\omega=q v, q$ is the phonon wave vector and $v$ is the phonon propagation velocity, $T$ is the temperature, $k_{\mathrm{B}}$ is the Boltzmann constant. More details can be found in the Supplementary Information Eq. (22), where a plot of the temperature dependence of the relaxation rate is presented as well. For $T=4 \mathrm{~K}$, the relaxation time is $17 \mathrm{~ms}$, suggesting that the qubit can easily be operated at this temperature.

\section{Applicability and implementation}

Although we have used a simple parabolic model for the in-plane QD confinement, our conclusions are very general. Firstly, the dephasing optimal operation point will be present for potentials of arbitrary complexity (for example hut wire geometries) ${ }^{7,55,56}$, since it is due to the fundamental interplay between the $\mathrm{HH}$ and $\mathrm{LH}$ that gives rise to the Rashba spin-orbit coupling in the $\mathrm{HH}$ manifold. Secondly, we have examined the possibility that the insensitivity of the $g$-factor to in-plane electric fields is an artifact of the model. We have tested three deviations from parabolicity and found that none of them exposes the qubit to dephasing by fluctuating in-plane electric fields. This implies (i) that the dot does not have to be perfectly parabolic allowing for some flexibility in the gate structure; (ii) that in-plane electric field fluctuations generally have a negligible effect on the $g$ factor, while out-of-plane electric field fluctuations cause fluctuations in the Rashba spin-orbit coupling and affect the $g$-factor, therefore it is most important to avoid the effect of the out-of-plane field; and (iii) that dephasing at the optimal operation point itself comes about primarily from higher-order terms in the electrical potential, i.e., electrical quadrupole and higher. Our results hold qualitatively in $\mathrm{Si}$ as well, where the spin-orbit interaction is weaker than in Ge, while $\delta$ is larger. However, the large $\delta$ and frequent failure of the SchriefferWolff approximation in Si calls for fully numerical treatment ${ }^{48}$.

Experimentally, the configuration we describe requires a double-gated device with separate plunger gates and barrier gates allowing the number density and the gate electric-field (and spin-orbit coupling) to be controlled independently ${ }^{58}$. The numerical estimates above suggest that, in general, a smooth and broad optimal operation point will enable the Ge hole qubit to work insensitively to the charge noise inside a large range of gate electric fields accessible to experiment. Exchange-based twoqubit gates should be possible for hole QDs, and their speed depends on the values of exchange obtained, which are expected to be tunable by gates. Moreover, it is likely to simplify the coupling between the two qubits since the valley degree of freedom is absent in hole systems. However, a two-qubit gate in the setup discussed here is not optimized for long-distance coupling, which leads to the two-qubit gate time is of the order of microseconds for dipole-dipole interactions and hundreds of microseconds for circuit QED, limited by the Ge Luttinger parameters. They can be sped up by enhancing the spin-orbit interaction, but we defer the discussion to a future publication.
A smaller $g$-factor will lead to a smaller Rabi frequency, a smaller change in the qubit Zeeman splitting due to the spinorbit interaction, and a shorter dephasing time but a longer relaxation time and an improved Rabi ratio. The optimal operation point will not change its location, which is determined only by the effective mass and the width of the quantum well. A larger quantum dot radius would make the confinement energy smaller, increasing the effect of the spin-orbit interaction and resulting in a faster Rabi frequency, but also shorter $T_{1}$ and $T_{2}{ }^{*}$. Nevertheless, the Rabi ratio decreases with increasing dot radius. Moreover, since the confinement energy decreases as the square of the radius, it is preferable to work at smaller radii to ensure the thermal broadening is overcome. Increasing $x$ increases the heavy hole - light hole splitting, leading to a reduced Rashba spin-orbit coupling and a smaller change in the qubit Zeeman splitting. The change in the Zeeman splitting will be large for smaller $x$ e.g., $0.05-0.10$, while at $x=0.3$ it is essentially not noticeable.

We have demonstrated that electrostatically defined hole quantum dot spin qubits naturally exhibit an optimal operation point at which sensitivity to charge noise is minimized while the speed of electrical operation is maximized. The location of the optimal operation point can be determined from the width of the quantum well and the strain tensors applied. Relaxation times are long even at $4 \mathrm{~K}$, while dephasing is determined by higher-order terms in the expansion of the electrostatic potential due to charge defects, but are expected to allow for a large window of operation around the optimal operation point. Our results provide a theoretical guideline for achieving fast, highly coherent, low-power electrically operated spin qubits experimentally. Future studies must consider in-plane magnetic fields, which interact much more weakly with $\mathrm{HH}$ spins and are more complicated to treat theoretically.

\section{METHODS}

\section{Numerical diagonalization}

Most of the results are obtained by theoretical analysis, we also present a numerical simulation by diagonalizing the full Hamiltonian. The full Hamiltonian is projected onto all the heavy-hole states and light-hole states and all the Fock-Darwin state.

\section{DATA AVAILABILITY}

Data sharing not applicable to this article as no data sets were generated or analyzed during this study.

Received: 19 December 2019; Accepted: 4 February 2021; Published online: 01 April 2021

\section{REFERENCES}

1. Loss, D. \& DiVincenzo, D. P. Quantum computation with quantum dots. Phys. Rev. A 57, 120-126 (1998).

2. Fischer, J., Trif, M., Coish, W. \& Loss, D. Spin interactions, relaxation and decoherence in quantum dots. Solid State Commun. 149, 1443 - 1450 (2009).

3. Chesi, S., Wang, X. J. \& Coish, W. A. Controlling hole spins in quantum dots and wells. Eur. Phys. J. Plus 129, 86 (2014).

4. Lodari, M. et al. Light effective hole mass in undoped $\mathrm{Ge} / \mathrm{SiGe}$ quantum wells. Phys. Rev. B 100, 041304 (2019).

5. Liles, S. D. et al. Spin and orbital structure of the first six holes in a silicon metaloxide-semiconductor quantum dot. Nat. Commun. 9, 3255 (2018).

6. Watzinger, H. et al. A germanium hole spin qubit. Nat. Commun. 9, 3902 (2018).

7. Watzinger, $\mathrm{H}$. et al. Heavy-hole states in germanium hut wires. Nano Lett. 16, 6879-6885 (2016).

8. Vukušić, L. et al. Single-shot readout of hole spins in Ge. Nano Lett. 18, 7141-7145 (2018).

9. Ares, N. et al. Sige quantum dots for fast hole spin Rabi oscillations. Appl. Phys. Lett. 103, 263113 (2013). 
10. Mizokuchi, R., Maurand, R., Vigneau, F., Myronov, M. \& De Franceschi, S. Ballistic one-dimensional holes with strong g-factor anisotropy in germanium. Nano Lett. 18, 4861-4865 (2018)

11. Bohuslavskyi, H. et al. Pauli blockade in a few-hole PMOS double quantum dot limited by spin-orbit interaction. Appl. Phys. Lett. 109, 193101 (2016).

12. Zwanenburg, F. A., van Rijmenam, C. E. W. M., Fang, Y., Lieber, C. M. \& Kou wenhoven, L. P. Spin states of the first four holes in a silicon nanowire quantum dot. Nano Lett. 9, 1071-1079 (2009).

13. Spruijtenburg, P. C. et al. Single-hole tunneling through a two-dimensional hole gas in intrinsic silicon. Appl. Phys. Lett. 102, 192105 (2013).

14. Brauns, M. et al. Anisotropic Pauli spin blockade in hole quantum dots. Phys. Rev. B 94, 041411 (2016).

15. Salfi, J., Mol, J. A., Culcer, D. \& Rogge, S. Charge-insensitive single-atom spin-orbit qubit in silicon. Phys. Rev. Lett. 116, 246801 (2016).

16. Salfi, J., Tong, M., Rogge, S. \& Culcer, D. Quantum computing with acceptor spins in silicon. Nanotechnology 27, 244001 (2016).

17. Hung, J.-T., Marcellina, E., Wang, B., Hamilton, A. R. \& Culcer, D. Spin blockade in hole quantum dots: Tuning exchange electrically and probing Zeeman interactions. Phys. Rev. B 95, 195316 (2017).

18. Terrazos, L. A. et al. Qubits Based on Hole Quantum Dots in Strained Ge. Phys. Rev. B Preprint at http://arxiv.org/abs/1803.10320. (2018).

19. van der Heijden, J. et al. Readout and control of the spin-orbit states of two coupled acceptor atoms in a silicon transistor. Sci. Adv. 4, eaat9199 (2018).

20. Chekhovich, E. A. et al. Nuclear spin effects in semiconductor quantum dots. Nat Mater. 12, 494 EP - (2013).

21. Kyrychenko, F. V. \& Kossut, J. Diluted magnetic semiconductor quantum dots: an extreme sensitivity of the hole Zeeman splitting on the aspect ratio of the confining potential. Phys. Rev. B 70, 205317 (2004)

22. Nadj-Perge, S., Frolov, S. M., Bakkers, E. P. A. M. \& Kouwenhoven, L. P. Spin-orbit qubit in a semiconductor nanowire. Nature 468, 1084 EP (2010).

23. Golovach, V. N., Khaetskii, A. \& Loss, D. Spin relaxation at the singlet-triplet crossing in a quantum dot. Phys. Rev. B 77, 045328 (2008).

24. Rossi, A. et al. Detection of variable tunneling rates in silicon quantum dots. Appl. Phys. Lett. 98, 133506 (2011).

25. Yoneda, J. et al. A quantum-dot spin qubit with coherence limited by charge noise and fidelity higher than 99.9\%. Nat. Nanotechnol. 13, 102-106 (2018).

26. Stano, P. et al. Orbital effects of a strong in-plane magnetic field on a gatedefined quantum dot. Phys. Rev. B 99, 085308 (2019).

27. Bulaev, D. V. \& Loss, D. Electric dipole spin resonance for heavy holes in quantum dots. Phys. Rev. Lett. 98, 097202 (2007).

28. Golovach, V. N., Borhani, M. \& Loss, D. Electric-dipole-induced spin resonance in quantum dots. Phys. Rev. B 74, 165319 (2006).

29. Coish, W. A., Golovach, V. N., Egues, J. C. \& Loss, D. Measurement, control, and decay of quantum-dot spins. Phys. Status Solid. 243, 3658-3672 (2006).

30. Szumniak, P., Bednarek, S., Pawłowski, J. \& Partoens, B. All-electrical control of quantum gates for single heavy-hole spin qubits. Phys. Rev. B 87, 195307 (2013).

31. Budich, J. C., Rothe, D. G., Hankiewicz, E. M. \& Trauzettel, B. All-electric qubit control in heavy hole quantum dots via non-abelian geometric phases. Phys. Rev. B 85, 205425 (2012).

32. Salis, G. et al. Electrical control of spin coherence in semiconductor nanostructures. Nature 414, 619-622 (2001).

33. Pribiag, V. S. et al. Electrical control of single hole spins in nanowire quantum dots. Nat. Nanotechnol. 8, 170 EP - (2013).

34. Khaetskii, A. V. Spin relaxation in semiconductor mesoscopic systems. Phys. E10 $27-31$ (2001)

35. Gvozdić, D. M. \& Ekenberg, U. Superiority of p-type spin transistors. Phys. Scripta T126, 21-26 (2006)

36. Woods, L. M., Reinecke, T. L. \& Kotlyar, R. Hole spin relaxation in quantum dots. Phys. Rev. B 69, 125330 (2004)

37. Hsieh, C.-Y., Cheriton, R., Korkusinski, M. \& Hawrylak, P. Valence holes as Luttinger spinor based qubits in quantum dots. Phys. Rev. B 80, 235320 (2009).

38. Kloeffel, C., Trif, M. \& Loss, D. Strong spin-orbit interaction and helical hole states in Ge/Si nanowires. Phys. Rev. B 84, 195314 (2011).

39. Machnikowski, P., Gawarecki, K. \& Cywiński, L. Hyperfine interaction for holes in quantum dots: $k \cdot p$ model. Phys. Rev. B 100, 085305 (2019).

40. Szumniak, P., Bednarek, S., Partoens, B. \& Peeters, F. M. Spin-orbit-mediated manipulation of heavy-hole spin qubits in gated semiconductor nanodevices. Phys. Rev. Lett. 109, 107201 (2012).

41. Khaetskii, A. V. \& Nazarov, Y. V. Spin relaxation in semiconductor quantum dots. Phys. Rev. B 61, 12639-12642 (2000).

42. Khaetskii, A. V., Loss, D. \& Glazman, L. Electron spin decoherence in quantum dots due to interaction with nuclei. Phys. Rev. Lett. 88, 186802 (2002).

43. Fischer, J. \& Loss, D. Hybridization and spin decoherence in heavy-hole quantum dots. Phys. Rev. Lett. 105, 266603 (2010)
44. Kobayashi, T. et al. Engineering long spin coherence times of spin-orbit qubits in silicon. Nat. Mater. 20, 38-42, https://doi.org/10.1038/s41563-020-0743-3 (2021).

45. Yamahata, G. et al. Magnetic field dependence of Pauli spin blockade: a window into the sources of spin relaxation in silicon quantum dots. Phys. Rev. B 86, 115322 (2012).

46. Hendrickx, N. W., Franke, D. P., Sammak, A., Scappucci, G. \& Veldhorst, M. Fast two-qubit logic with holes in germanium. Nature 577, 487 (2020).

47. Durnev, M. V., Glazov, M. M. \& Ivchenko, E. L. Spin-orbit splitting of valence subbands in semiconductor nanostructures. Phys. Rev. B 89, 075430 (2014).

48. Marcellina, E., Hamilton, A. R., Winkler, R. \& Culcer, D. Spin-orbit interactions in inversion-asymmetric two-dimensional hole systems: A variational analysis. Phys. Rev. B 95, 075305 (2017).

49. Bermeister, A., Keith, D. \& Culcer, D. Charge noise, spin-orbit coupling, and dephasing of single-spin qubits. Appl. Phys. Lett. 105, 192102 (2014).

50. Culcer, D., Hu, X. \& Das Sarma, S. Dephasing of si spin qubits due to charge noise Appl. Phys. Lett. 95, 073102 (2009).

51. Fischer, J., Coish, W. A., Bulaev, D. V. \& Loss, D. Spin decoherence of a heavy hole coupled to nuclear spins in a quantum dot. Phys. Rev. B 78, 155329 (2008).

52. Csontos, D., Brusheim, P., Zülicke, U. \& Xu, H. Q. Spin- $\frac{3}{2}$ physics of semiconductor hole nanowires: valence-band mixing and tunable interplay between bulkmaterial and orbital bound-state spin splittings. Phys. Rev. B 79, 155323 (2009).

53. Meier, L. et al. Measurement of Rashba and Dresselhaus spin-orbit magnetic fields. Nat. Phys. 3, 650 EP - (2007).

54. Governale, M. \& Zülicke, U. Spin accumulation in quantum wires with strong Rashba spin-orbit coupling. Phys. Rev. B 66, 073311 (2002).

55. Li, S.-X. et al. Measuring hole spin states of single quantum dot in germanium hut wire. Appl. Phys. Lett. 110, 133105 (2017).

56. Li, Y. et al. Coupling a germanium hut wire hole quantum dot to a superconducting microwave resonator. Nano Lett. 18, 2091-2097 (2018).

57. Kloeffel, C., Trif, M., Stano, P. \& Loss, D. Circuit QED with hole-spin qubits in Ge/Si nanowire quantum dots. Phys. Rev. B 88, 241405 (2013).

58. Sammak, A. et al. Shallow and undoped germanium quantum wells: a playground for spin and hybrid quantum technology. Adv. Funct. Mater. 29, 1807613 (2019).

59. Hardy, W. J. et al. Single and double hole quantum dots in strained Ge/SiGe quantum wells. Nanotechnology 30, 215202 (2019).

60. Takeda, K. et al. Characterization and suppression of low-frequency noise in $\mathrm{Si} /$ SiGe quantum point contacts and quantum dots. Appl. Phys. Lett. 102, 123113 (2013).

61. Katsaros, G. et al. Observation of spin-selective tunneling in SiGe nanocrystals Phys. Rev. Lett. 107, 246601 (2011).

62. Rol, M. et al. Fast, high-fidelity conditional-phase gate exploiting leakage interference in weakly anharmonic superconducting qubits. Phys. Rev. Lett. 123 https://doi.org/10.1103/PhysRevLett.123.120502. (2019).

63. Luttinger, J. M. \& Kohn, W. Motion of electrons and holes in perturbed periodic fields. Phys. Rev. 97, 869-883 (1955)

64. Marcellina, E. et al. Electrical control of the Zeeman spin splitting in twodimensional hole systems. Phys. Rev. Lett. 121, 077701 (2018).

65. Liu, H., Marcellina, E., Hamilton, A. R. \& Culcer, D. Strong spin-orbit contribution to the hall coefficient of two-dimensional hole systems. Phys. Rev. Lett. 121, 087701 (2018).

66. Bastard, G., Mendez, E. E., Chang, L. L. \& Esaki, L. Variational calculations on a quantum well in an electric field. Phys. Rev. B 28, 3241-3245 (1983).

67. Golovach, V. N., Khaetskii, A. \& Loss, D. Phonon-induced decay of the electron spin in quantum dots. Phys. Rev. Lett. 93, 016601 (2004).

68. Trif, M., Simon, P. \& Loss, D. Relaxation of hole spins in quantum dots via twophonon processes. Phys. Rev. Lett. 103, 106601 (2009).

69. Bulaev, D. V. \& Loss, D. Spin relaxation and decoherence of holes in quantum dots. Phys. Rev. Lett. 95, 076805 (2005).

70. Maier, F., Kloeffel, C. \& Loss, D. Tunable $g$ factor and phonon-mediated hole spin relaxation in Ge/Si nanowire quantum dots. Phys. Rev. B 87, 161305 (2013).

71. Woods, L. M., Reinecke, T. L. \& Lyanda-Geller, Y. Spin relaxation in quantum dots. Phys. Rev. B 66, 161318 (2002).

72. Climente, J. I., Segarra, C. \& Planelles, J. Spin-orbit-induced hole spin relaxation in InAs and GaAs quantum dots. New Journal of Physics 15, 093009 (2013).

73. Kornich, V., Kloeffel, C. \& Loss, D. Phonon-mediated decay of singlet-triplet qubits in double quantum dots. Phys. Rev. B 89, 085410 (2014).

74. Ares, N. et al. Nature of tunable hole $g$ factors in quantum dots. Phys. Rev. Lett. 110, 046602 (2013)

75. Laucht, A. et al. Electrically controlling single-spin qubits in a continuous microwave field. Sci. Adv. 1, e1500022 (2015).

76. Li, R., Hudson, F. E., Dzurak, A. S. \& Hamilton, A. R. Pauli spin blockade of heavy holes in a silicon double quantum dot. Nano Lett. 15, 7314-7318 (2015).

77. Bravyi, S., DiVincenzo, D. P. \& Loss, D. Schrieffer-Wolff transformation for quantum many-body systems. Ann. Phys. 326, 2793 - 2826 (2011) 
78. Winkler, R. Rashba spin splitting in two-dimensional electron and hole systems. Phys. Rev. B 62, 4245-4248 (2000).

79. Winkler, R., Papadakis, S. J., De Poortere, E. P. \& Shayegan, M. Highly anisotropic $g$ factor of two-dimensional hole systems. Phys. Rev. Lett. 85, 4574-4577 (2000).

80. Habib, B. et al. Negative differential Rashba effect in two-dimensional hole systems. Appl. Phys. Lett. 85, 3151-3153 (2004).

81. Press, D. et al. Ultrafast optical spin echo in a single quantum dot. Nat. Photon. 4 , 367 (2010).

\section{ACKNOWLEDGEMENTS}

We thank Mark Friesen and András Pályi for a multitude of engaging and educational discussions. We are also grateful to Thaddeus Ladd, Sue Coppersmith, and Andrè Saraiva for stimulating feedback. This research is supported by the Australian Research Council Centre of Excellence in Future Low-Energy Electronics Technologies (project CE170100039) and funded by the Australian Government.

\section{AUTHOR CONTRIBUTIONS}

Z.W. performed all the calculations presented in this work in collaboration with E.M. J.H.C. assisted with the theoretical calculations. J.S., A.H., and S.R. contributed to the discussion of experimental implementation. D.C. and J.S. devised the theoretical model, supervised the calculations, and wrote the manuscript.

\section{COMPETING INTERESTS}

The authors declare no competing interests.

\section{ADDITIONAL INFORMATION}

Supplementary information The online version contains supplementary material available at https://doi.org/10.1038/s41534-021-00386-2.

Correspondence and requests for materials should be addressed to Z.W. or D.C.

Reprints and permission information is available at http://www.nature.com/ reprints

Publisher's note Springer Nature remains neutral with regard to jurisdictional claims in published maps and institutional affiliations.

(i) Open Access This article is licensed under a Creative Commons C. Attribution 4.0 International License, which permits use, sharing, adaptation, distribution and reproduction in any medium or format, as long as you give appropriate credit to the original author(s) and the source, provide a link to the Creative Commons license, and indicate if changes were made. The images or other third party material in this article are included in the article's Creative Commons license, unless indicated otherwise in a credit line to the material. If material is not included in the article's Creative Commons license and your intended use is not permitted by statutory regulation or exceeds the permitted use, you will need to obtain permission directly from the copyright holder. To view a copy of this license, visit http://creativecommons. org/licenses/by/4.0/.

(c) The Author(s) 2021 